\title{
MULTIPLIERS OF CLOSED IDEALS IN GROUP ALGEBRAS
}

\author{
STEPHEN H. FRIEDBERG
}

\begin{abstract}
The purpose of this paper is to show that the multipliers of a special class of closed ideals in group algebras are "trivial", and that a result of $\mathrm{Y}$. Meyer for groups of the form $R^{n} \times T^{m}$ concerning the existence of nontrivial multipliers cannot be extended to any disconnected group.
\end{abstract}

The following notation will be used throughout this paper and is essentially the same as that used in [3] and [4]. $G$ will denote a locally compact abelian group (lcag) with dual group $\Gamma . L^{1}(G)$ and $M(G)$ will denote the group algebra and measure algebra of $G$ with transform algebras $A(\Gamma)$ and $B(\Gamma)$, respectively. The norm in $A(\Gamma)$ will be the induced norm from $L^{1}(G)$, that is $\left\|f^{\wedge}\right\|=\|f\|_{1}$. Finally, $\Gamma_{d}$ will denote the group $\Gamma$ with the discrete topology.

For what follows, $I$ will be a closed nonzero ideal of $L^{1}(G)$ with zero set $E$. A complex-valued function $\phi$ on $\Gamma$ is a multiplier of $I$ if $\phi f^{\wedge} \in A(\Gamma)$ for every $f \in I$ and, for convenience, $\phi=0$ on $E$. By the closed graph theorem the map $f^{\wedge} \rightarrow \phi f^{\wedge}$ from $I^{\wedge}$ to $A(\Gamma)$ is continuous, and hence $\phi$ is a continuous function on $E^{\prime}$, the complement of $E$. $\phi$ is said to be trivial if there exists $\mu \in M(G)$ such that $\phi=\mu^{\wedge}$ on $E^{\prime}$. In particular, $\phi$ is trivial if $\phi \in B(\Gamma)$.

Meyer [3, p. 521] has shown that if $\Gamma=R^{n} \times T^{m}$, where $R$ and $T$ denote the real line and circle, respectively, then there exists a nontrivial multiplier of $I$ if and only if the interior of $E$ is not empty. We will prove in Theorem 2 that in fact Meyer's result is false if $\Gamma$ is any disconnected group.

THEOREM 1. Let $G$ be a lcag with dual group $\Gamma$ and let $E \neq \Gamma$ be any element of the coset ring of $\Gamma_{d}$ (see, for example, [4, p. 60]). If I is any closed ideal of $L^{1}(G)$ with zero set $E$, then every multiplier of $I$ is trivial.

Received by the editors March 7, 1973.

AMS (MOS) subject classifications (1970). Primary 43A25; Secondary 43A18.

Key words and phrases. Locally compact abelian group, dual group, group algebra, measure algebra, multiplier, coset ring, Bohr compactification, Eberlein's theorem, Cohen's idempotent theorem.

(c) American Mathematical Society 1973 
Proof. Let $\phi$ be a multiplier of $I$. It follows from Meyer [3, p. 504] that $\phi$ is a multiplier of $I^{\sim}=\left\{f \in L^{1}(\bar{G}): f^{\wedge}=0\right.$ on $\left.E\right\}$ where $\bar{G}$ denotes the Bohr compactification of $G$. Since $E$ belongs to the coset ring of $\Gamma_{d}$, we may use Cohen's idempotent theorem $[1]$ or $[4$, p. 60] to obtain $\lambda \in M(\bar{G})$ such that $\lambda^{\wedge}$ is the characteristic function of $E^{\prime}$. Let $\left\{u_{\alpha}\right\}$ be a bounded approximate identity of trigonometric polynomials for $L^{1}(\bar{G})$, then $\left\{u_{\alpha} * \lambda\right\}$ is clearly a bounded approximate identity of trigonometric polynomials for $I^{\sim}$. Now, as we observed earlier, the map $f^{\wedge} \rightarrow \phi f^{\wedge}$ is continuous on $I^{\wedge}$ so we have that $\sup _{\alpha}\left\|\phi u_{a}^{\hat{\lambda}} \lambda^{\wedge}\right\|<\infty$. Also, for each $\gamma \in \Gamma$, $\left(\phi u_{\alpha}^{\hat{\lambda}} \lambda^{\wedge}\right)(\gamma) \rightarrow \phi(\gamma)$. Therefore, by Eberlein's theorem [4, p. 32] we may deduce that $\phi \in B\left(\Gamma_{d}\right)$. But $\phi$ is continuous on $E^{\prime}$ so from [2, p. 212] it follows that there exists $\mu \in M(G)$ such that $\mu^{\wedge}=\phi$ on $E^{\prime}$. Hence, $\phi$ is trivial.

THEOREM 2. If $\Gamma$ is not connected, then there exists a closed ideal $I$ of $L^{1}(G)$ with zero set $E$ such that $E$ has nonempty interior, yet every multiplier $\phi$ of $I$ is trivial. In fact, every such $\phi$ belongs to $B(\Gamma)$.

Proof. Since $\Gamma$ is not connected, we may deduce from the principal structure theorem for a lcag that there exists a proper open (hence closed) subgroup $E$ of $\Gamma$. Let $I$ be any closed ideal of $L^{1}(G)$ with zero set $E$, and let $\phi$ be a multiplier of $I$. By Theorem $1, \phi$ is trivial. However, since $\phi$ is continuous on all of $\Gamma$, the proof of Theorem 1 shows that in fact $\phi \in B(\Gamma)$.

REMARK. It should be noted that Theorem 2 can be proved directly without appealing to the results of either [1] or [2] since, in this case, $E$ is both open and closed.

\section{REFERENCES}

1. P. J. Cohen, On a conjecture of Littlewood and idempotent measures, Amer. J. Math. 82 (1960), 191-212. MR 24 \#A3231.

2. R. Doss, Approximations and representations for Fourier transforms, Trans. Amer. Math. Soc. 153 (1971), 211-221. MR 42 \#3494.

3. Y. Meyer, Endomorphismes des idéaux fermés de $L^{1}(G)$, classes de Hardy et séries de Fourier lacunaires, Ann. Sci. Ecole Norm. Sup. (4) 1 (1968), 499-580. MR 39 \#1910.

4. W. Rudin, Fourier analysis on groups, Interscience Tracts in Pure and Appl. Math., no. 12, Interscience, New York, 1962. MR 27 \#2808.

Department of Mathematics, Illinois State University, Normal, Illinois 61761 